\title{
Influence of Temperature on the Corrosion Potential of the 241-AN-102 Multi-Probe Corrosion Monitoring System Secondary Reference Electrodes
}

\author{
G. L. Edgemon and T. M. Taylor \\ ARES Corporation for CH2M HILL Hanford Group, Inc. \\ Richland, WA 99352 \\ U.S. Department of Energy Contract DE-AC27-99RL14047

$\begin{array}{lll}\text { EDT/ECN: } & \text { DRF } & \text { UC: N/A } \\ \text { Cost Center: } & \text { N/A } & \text { Charge Code: } \\ \text { B\&R Code: } & \text { N/A } & \text { Total Pages: } 2021 \\ & & \end{array}$

Key Words: 241-AN-102 Multi-Probe Corrosion Monitoring System, reference electrode testing, corrosion potential

\begin{abstract}
A test program using 241-AN-102 waste simulants and metallic secondary reference electrodes similar to those used on the 241-AN-102 MPCMS was performed to characterize the relationship between temperature and secondary reference electrode open-circuit corrosion potential. This program showed that the secondary reference electrodes can be used to make tank and tank steel corrosion potential measurements, but that a correction factor of approximately $2 \mathrm{mV}$ per degree Celsius of temperature difference must be applied, where temperature difference is defined as the difference between tank temperature at the time of measurement and $30^{\circ} \mathrm{C}$, the average tank temperature during the first several months of 241-AN-102 MPCMS operation (when the corrosion potentials of the secondary reference electrodes were being recorded relative to the primary reference electrodes).
\end{abstract}

TRADEMARK DISCLAIMER. Reference herein to any specific commercial product, process, or service by trade name, trademark, manufacturer, or otherwise, does not necessarily constitute or imply its endorsement, recommendation, or favoring by the United States Govemment or any agency thereof or its contractors or subcontractors.

Printed in the United States of America. To obtain copies of this document, contact: Document Control Services, P.O. Box 950, Mailstop H6-08, Richland WA 99352, Phone (509) 372-2420; Fax (509) 376-4989.

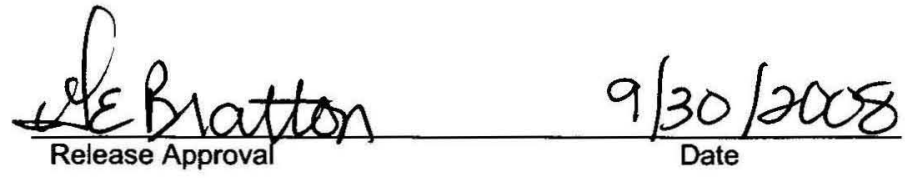

Approved For Public Release

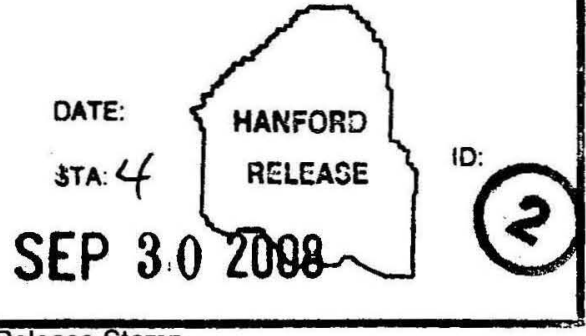

Release Stamp 


\section{Influence of Temperature on the Corrosion Potential of the 241-AN-102 Multi-Probe Corrosion Monitoring System Secondary Reference Electrodes}

G. L. Edgemon

T. M. Taylor

ARES Corporation

K. G. Carothers

CH2M HILL Hanford Group, Inc.

Date Published

September 2008

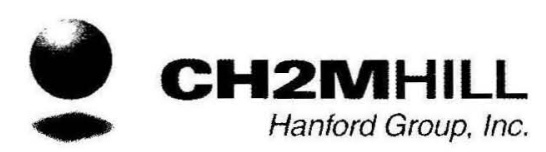

Post Office Box 1500

Richland, Washington

Prepared for the U.S. Department of Energy

Office of River Protection

Approved for public release; distribution unlimited 


\section{TABLE OF CONTENTS}

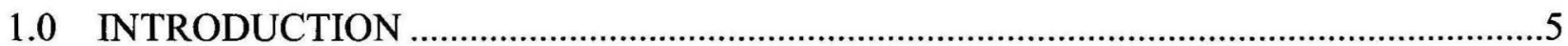

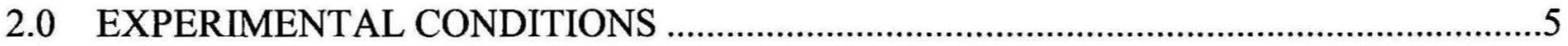

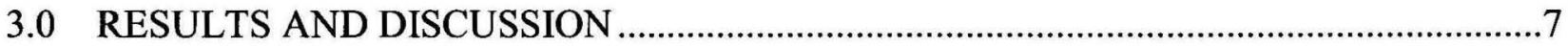

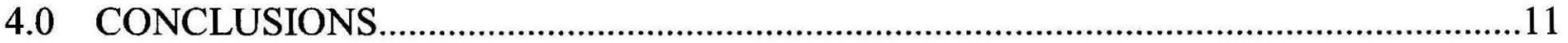

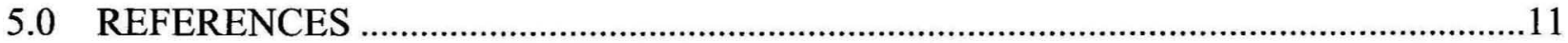

\section{LIST OF APPENDICES}

Appendix A - 241-AN-102 "Present" Simulant Composition

Appendix B - 241-AN-102 "10-Year" Simulant Composition

Appendix C - 241-AN-102 "20-Year" Simulant Composition

\section{LIST OF FIGURES}

Figure 2-1. Constant Temperature Bath and Test Cell Assembly. ..............................................

Figure 3-1. Corrosion Potential and Bath Temperature vs. Time, Copper Electrodes. .................8

Figure 3-2. Corrosion Potential and Bath Temperature vs. Time, Nickel Electrodes. ..................8

Figure 3-3. Corrosion Potential and Bath Temperature vs. Time, Silver Electrodes. ...................9

Figure 3-4. Corrosion Potential and Bath Temperature vs. Time ...............................................10

Figure 3-5. Temperature vs. Average Corrosion Potentials. ....................................................11

\section{LIST OF TABLES}

Table 2-1. Test Matrix. .6 
RPP-RPT-38931, Rev. 0

\section{LIST OF TERMS}

Abbreviations and Acronyms

MPCMS Multi-Probe Corrosion Monitoring System

SCE saturated calomel electrode

Units

$\begin{array}{ll}{ }^{\circ} \mathrm{C} & \text { Degrees Celsius } \\ \text { in. } & \text { inch } \\ \mathrm{M} & \text { Molarity } \\ \mathrm{L} & \text { Liters } \\ \% & \text { percent } \\ \mathrm{mV} & \text { millivolts }\end{array}$




\subsection{INTRODUCTION}

In May 2008, CH2M HILL Hanford Group, Inc. installed the first Multi-Probe Corrosion Monitoring System (MPCMS) in double-shell tank 241-AN-102. The 241-AN-102 MPCMS contains six, double-junction primary reference electrodes: two silver/silver chloride electrodes, two saturated calomel electrodes, and two silver chloride/copper sulfate electrodes. These primary electrodes are positioned on the MPCMS so that, after installation, one electrode of each type is located in the supernatant layer of the tank and one of each type is located in the saltcake layer of the tank. The primary reference electrodes are used to measure the corrosion potential of the tank and four tank steel electrodes mounted on the MPCMS.

Because the primary reference electrodes may ultimately fail, either due to electrolyte contamination or radiation damage, the 241-AN-102 MPCMS also contains six metallic, pintype secondary reference electrodes: two copper electrodes, two nickel electrodes, and two silver electrodes. These secondary electrodes are positioned on the MPCMS so that, after installation, one electrode of each type is located in the supernatant layer of the tank and one of each type is located in the saltcake layer of the tank. The corrosion potential of the secondary electrodes (and the tank and tank metal electrodes) will be measured using the primary reference electrodes until the primary electrodes fail. Following the failure of the primary electrodes, the corrosion potential of the tank and tank metal electrodes will be measured using the secondary reference electrodes. Additional details of the 241-AN-102 MPCMS design and operating requirements can be found in RPP-SPEC-32496, Procurement Specification for AN-102 Corrosion Probe Assembly.

As described in RPP-PLAN-24604, Test Plan for Evaluating Secondary Reference Electrode Materials for the 241-AN-102 and the 241-AY-102 Multi-Probe Corrosion Monitoring Systems, a series of laboratory tests were required to establish the effect of waste temperature on the corrosion potential of the copper, nickel, and silver secondary electrodes. The results of this test program are contained herein.

\subsection{EXPERIMENTAL CONDITIONS}

Copper, nickel, and silver electrodes were tested. Metals used for testing were at least $99.9 \%$ pure. Each electrode was formed from a 1/8-in. diameter rod, approximately 18 inches in length. Each electrode was coated in epoxy then shrink-wrapped to prevent corrosion of the material in the vapor space above the test solution. Approximately one inch of the bottom of each electrode was left exposed to provide contact with the test solution after immersion in the test cell. The top inch of each electrode was left exposed to facilitate electrical contact during corrosion potential measurements. Three nominally identical electrodes of each material were prepared.

Nine polyethylene test cells, each approximately $2 \mathrm{~L}$ in volume, were assembled. Each cell was fitted to hold one metallic electrode. Each test cell was also fitted with an Allihn-type watercooled condenser (to minimize the evaporation of cell contents), and a rubber stopper that could be temporarily removed to facilitate corrosion potential measurements. On February 28, 2008, each test cell was filled with a variation of the 241-AN-102 waste simulant (either "Present," "10-Year," or " 20 -Year" formulation) and loaded into a constant temperature bath set at $30^{\circ} \mathrm{C}$. 
Waste simulants used in testing were formulated to match the present waste chemistry in 241-AN-102 (i.e., "Present" simulant), plus projected waste chemistries 10 years and 20 years in the future. The test matrix is shown in Table 2-1. The 241-AN-102 "Present," "10-Year," and "20-Year" waste simulant formulations are shown in Appendices A, B, and C, respectively. Both target chemistries and formulations (as-mixed) are shown for each of the three chemistries. The completed test assembly is shown in Figure 2-1.

Table 2-1. Test Matrix.

\begin{tabular}{|c|l|}
\hline Cell & \multicolumn{1}{|c|}{ Test Cell Contents } \\
\hline 1 & Copper Electrode in "Present" Simulant \\
\hline 2 & Copper Electrode in "10-Year" Simulant \\
\hline 3 & Copper Electrode in "20-Year" Simulant \\
\hline 4 & Nickel Electrode in "Present" Simulant \\
\hline 5 & Nickel Electrode in "10-Year" Simulant \\
\hline 6 & Nickel Electrode in "20-Year" Simulant \\
\hline 7 & Silver Electrode in "Present" Simulant \\
\hline 8 & Silver Electrode in "10-Year" Simulant \\
\hline 9 & Silver Electrode in "20-Year" Simulant \\
\hline
\end{tabular}

For the duration of testing, the temperature of the bath was changed every seven days in a repeating pattern of $10^{\circ} \mathrm{C}$ increments between $30^{\circ} \mathrm{C}$ and $50^{\circ} \mathrm{C}$ (i.e., $30^{\circ} \mathrm{C}$ for seven days, $40^{\circ} \mathrm{C}$ for seven days, $50^{\circ} \mathrm{C}$ for seven days, $40^{\circ} \mathrm{C}$ for seven days, then back to $30^{\circ} \mathrm{C}$ for seven days at which point the pattern was repeated). The bath temperature equilibrated within an hour of each change in temperature set point. The open-circuit potential of each secondary reference electrode was measured with a calibrated FLUKE ${ }^{\circledR 1}$ Model 87 III digital multimeter approximately once per day relative to a double-junction saturated calomel reference electrode (Radiometer ${ }^{\circledR 2}$ Analytical Model XR110) and associated salt bridge (Radiometer Analytical Model AL120). The salt bridge was filled prior to testing with saturated potassium chloride solution. Water was added to the constant temperature bath occasionally to keep test cells submerged. Data collection was terminated and electrodes removed from the test cells on August 3, 2008.

\footnotetext{
${ }^{1}$ FLUKE is a registered trademark of Fluke Corporation, Everett, Washington.

${ }^{2}$ Radiometer is a registered trademark of Radiometer Medical ApS, a public limited company, Brønshøj, Denmark.
} 


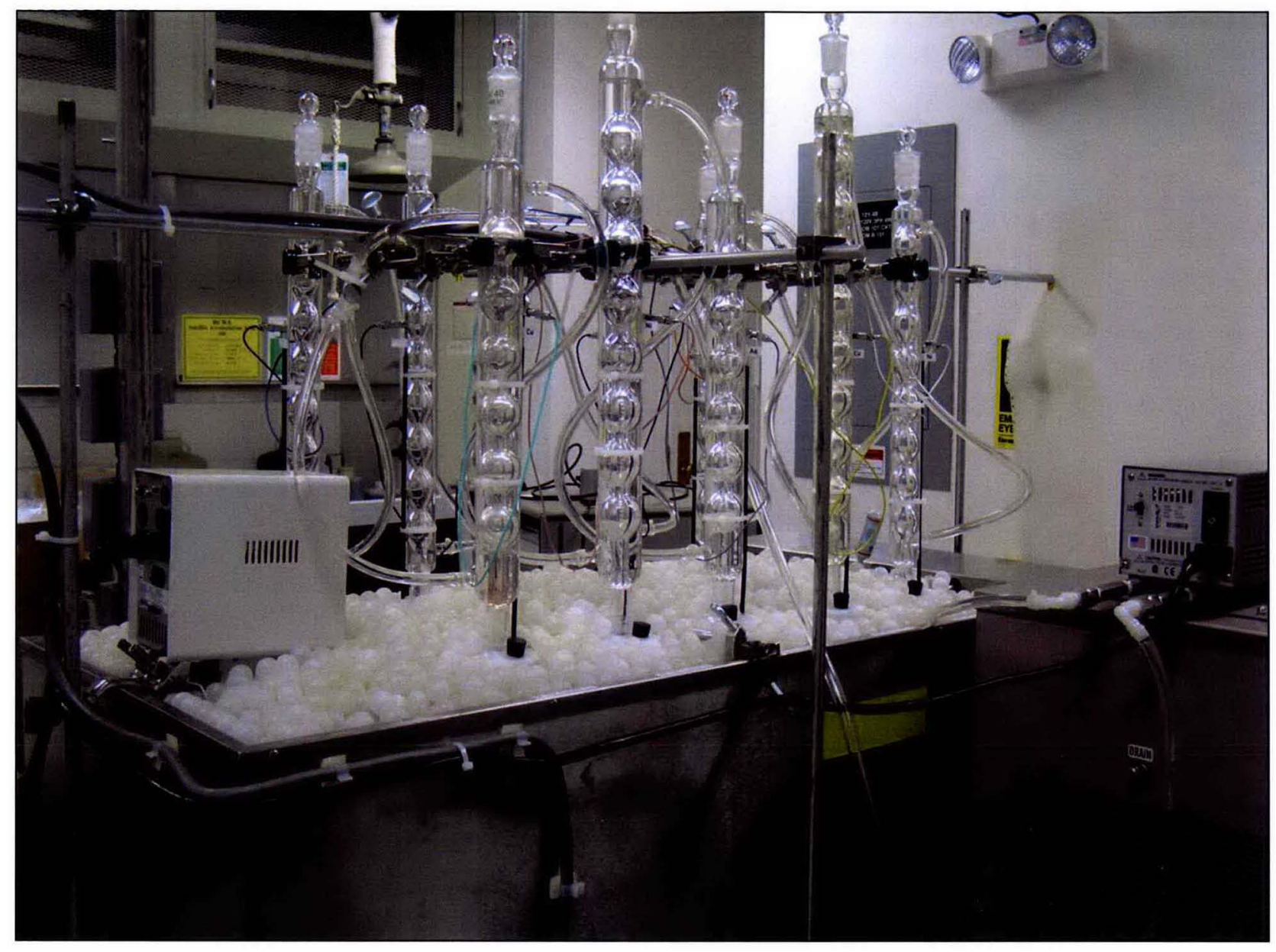

Figure 2-1. Constant Temperature Bath and Test Cell Assembly.

\subsection{RESULTS AND DISCUSSION}

Open circuit corrosion potential data for the copper, nickel, and silver secondary reference electrodes (relative to the saturated calomel electrode) are shown in Figures 3-1 through 3-3, respectively. 


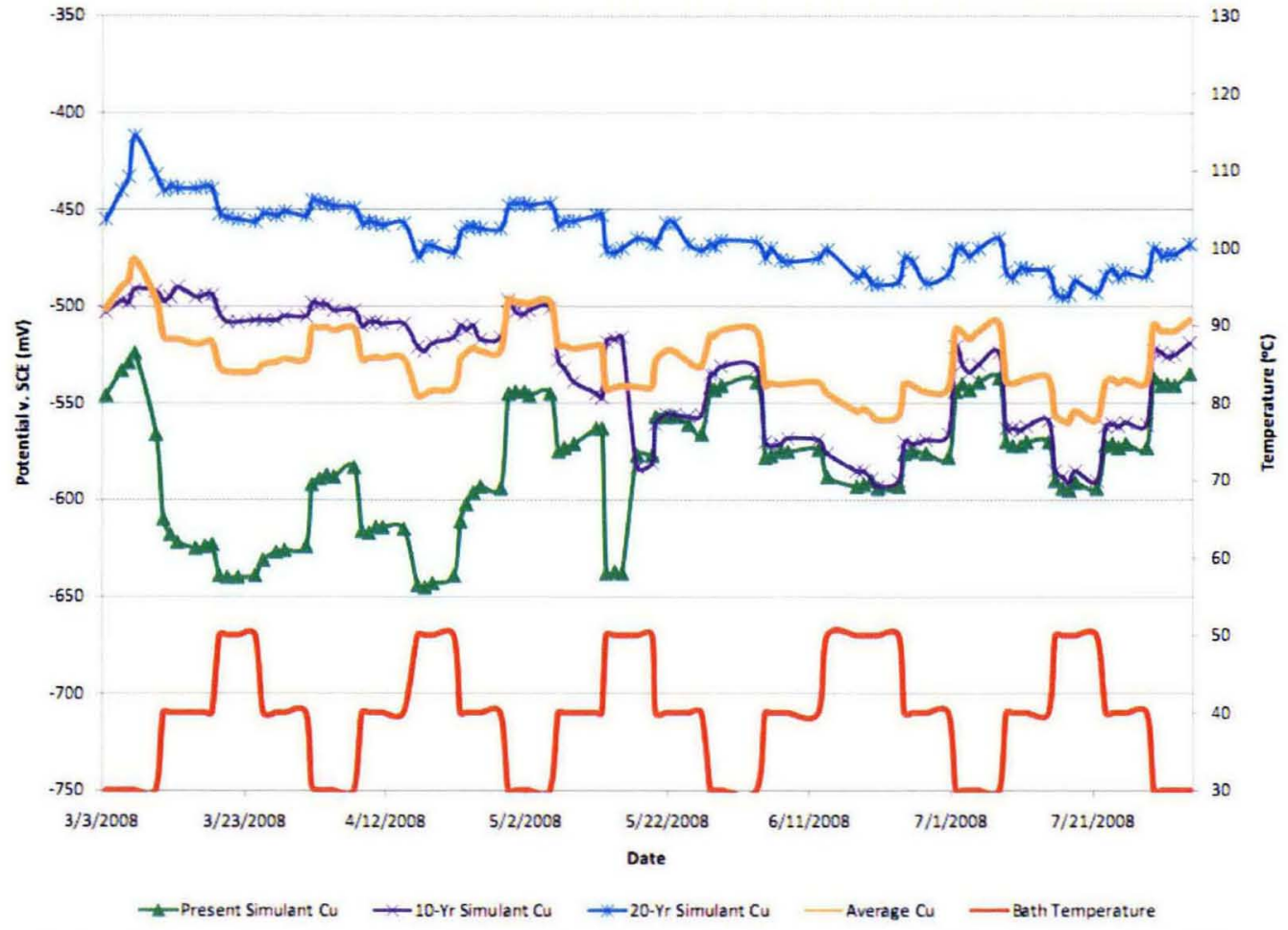

Figure 3-1. Corrosion Potential and Bath Temperature vs. Time, Copper Electrodes.

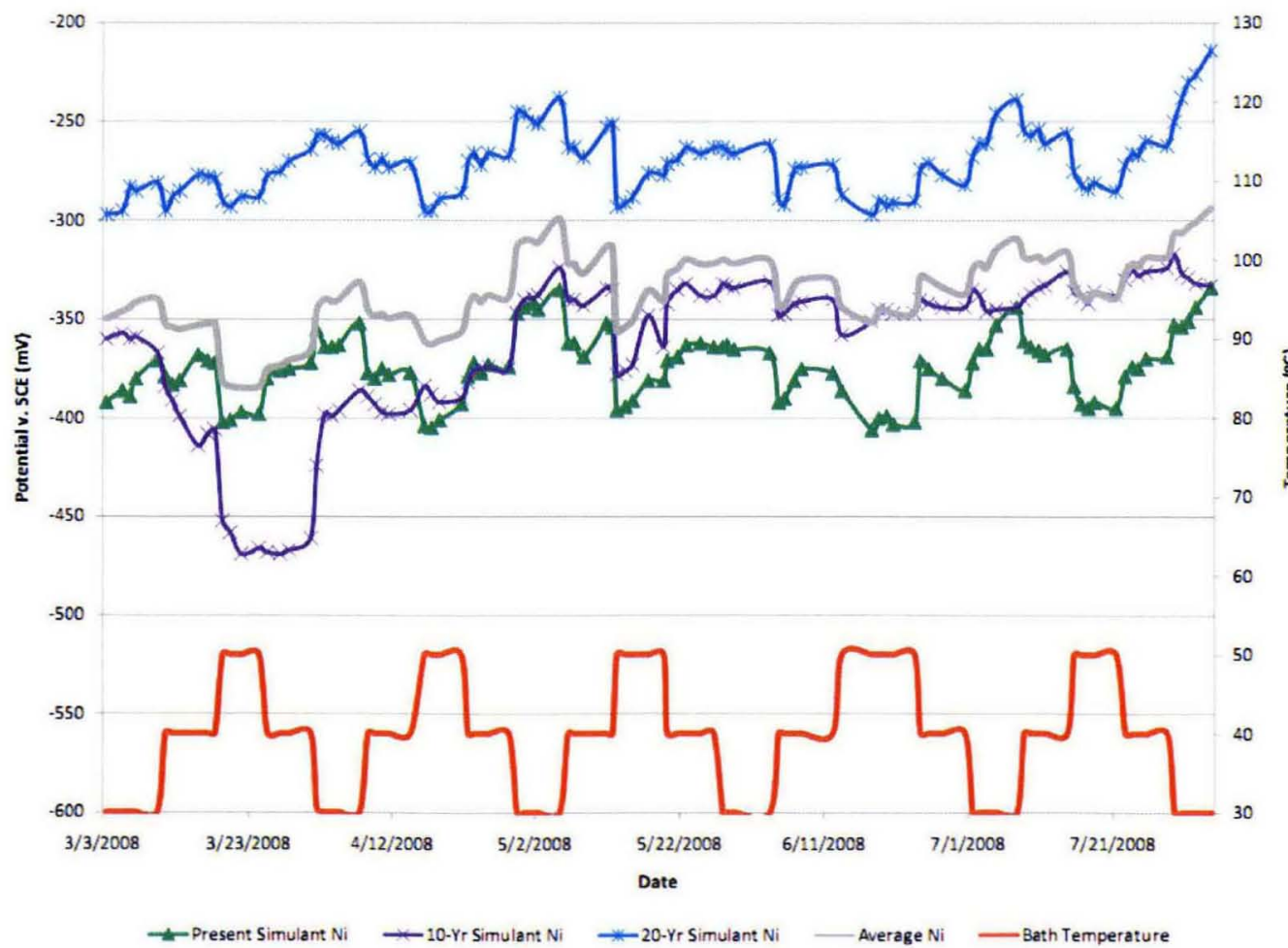

Figure 3-2. Corrosion Potential and Bath Temperature vs. Time, Nickel Electrodes. 


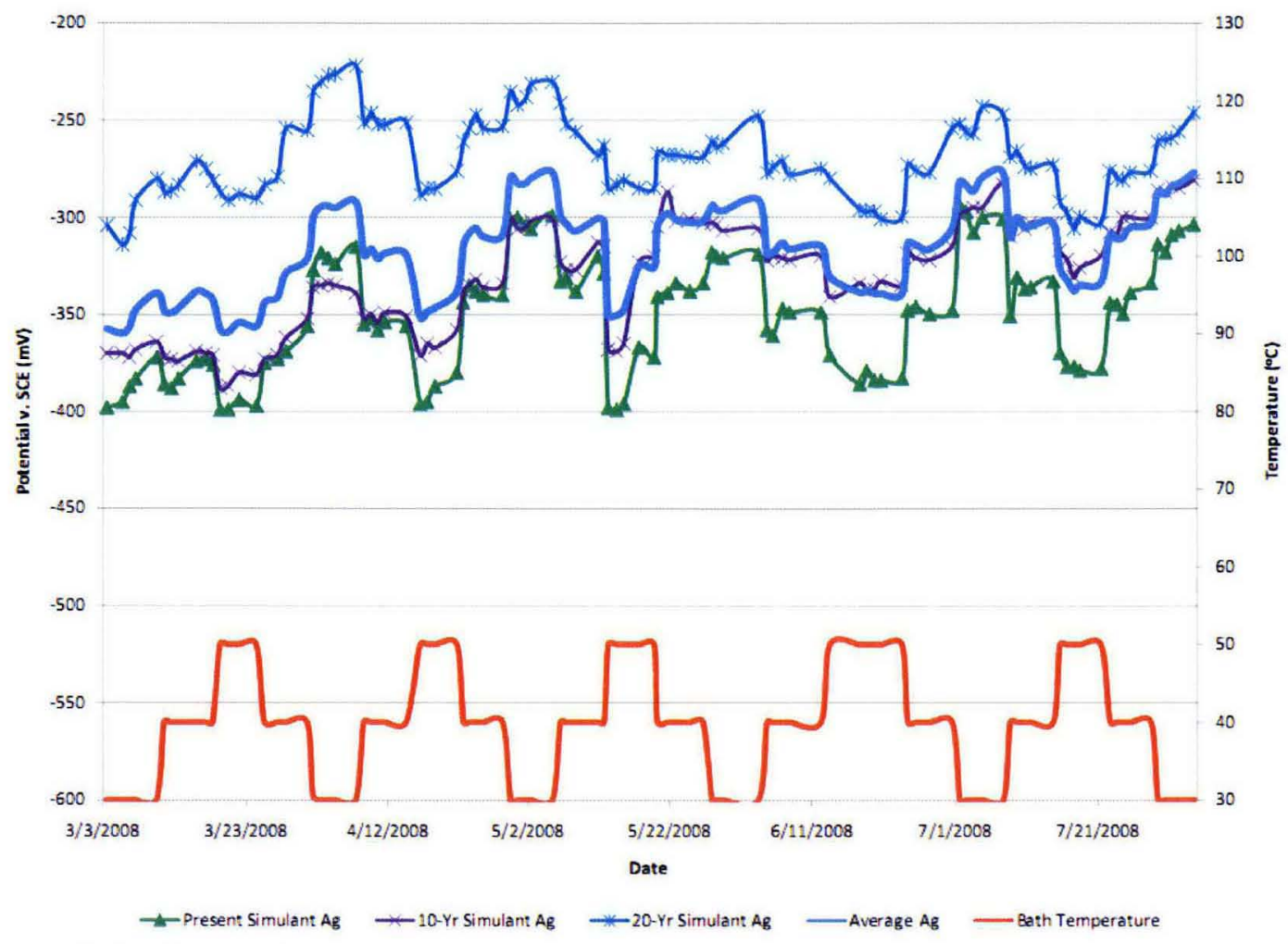

Figure 3-3. Corrosion Potential and Bath Temperature vs. Time, Silver Electrodes.

In general, the corrosion potential of the secondary electrodes changes inversely with temperature. For a given electrode material however, traditional statistical analyses identified no significant difference in the temperature/potential relationship between the three waste simulants. Because of this, and to minimize the impact of spurious noise and other sources of error, the results from the Present, 10-year, and 20-year simulants were averaged for each electrode material. The averaged corrosion potential and temperature readings are shown in Figure 3-4. 


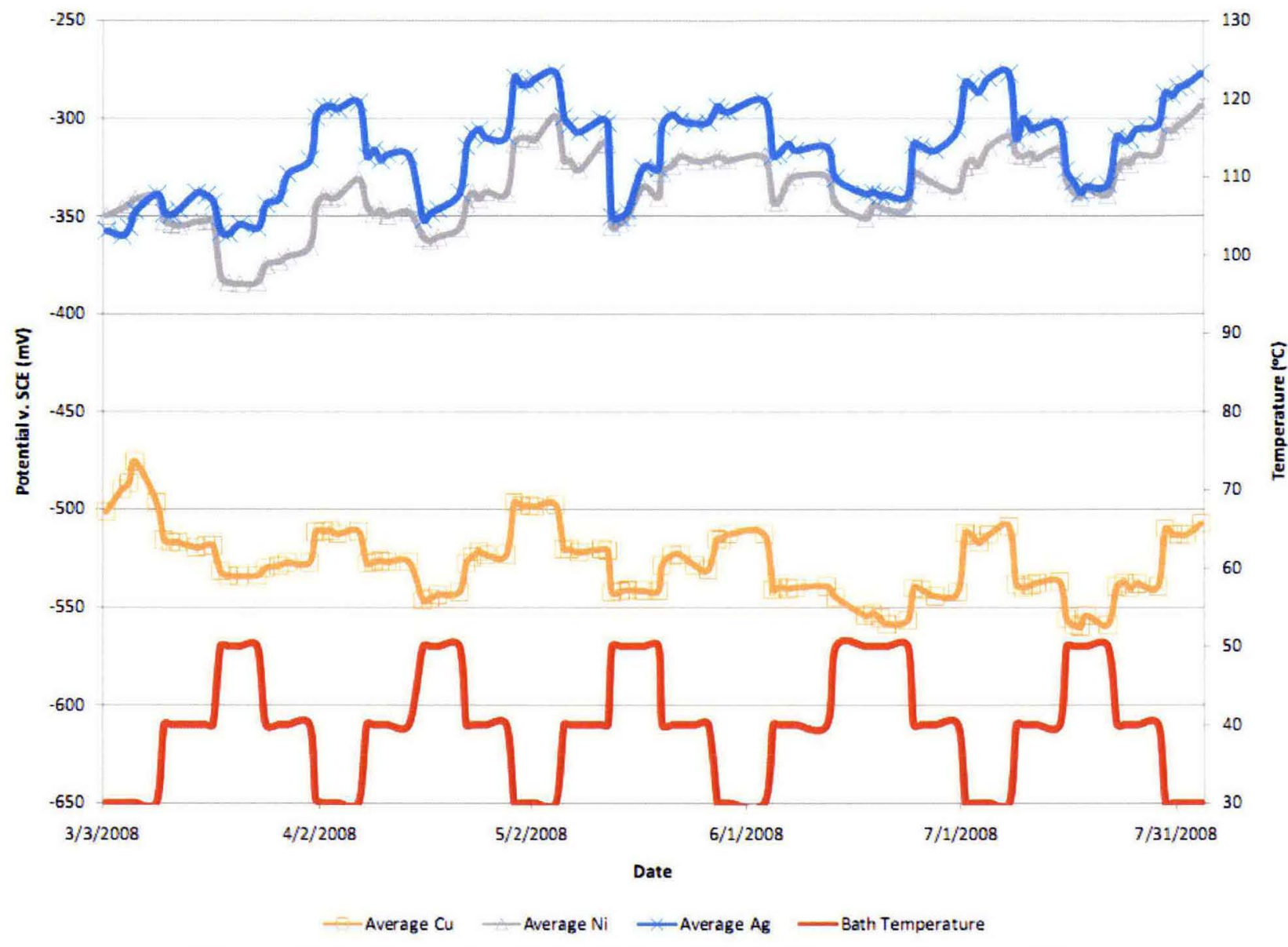

Figure 3-4. Corrosion Potential and Bath Temperature vs. Time.

The average difference in potential between the "high" $\left(50^{\circ} \mathrm{C}\right)$ and "low" $\left(30^{\circ} \mathrm{C}\right)$ temperature regions is approximately $40 \mathrm{mV}$ which equates to a shift in potential of approximately $2 \mathrm{mV}$ per degree Celsius. Performing a linear regression to determine the slope of each averaged set of potential data versus temperature, as seen in Figure 3-5, confirms these findings, displaying an average slope of $-2 \mathrm{mV}$ per degree Celsius. 


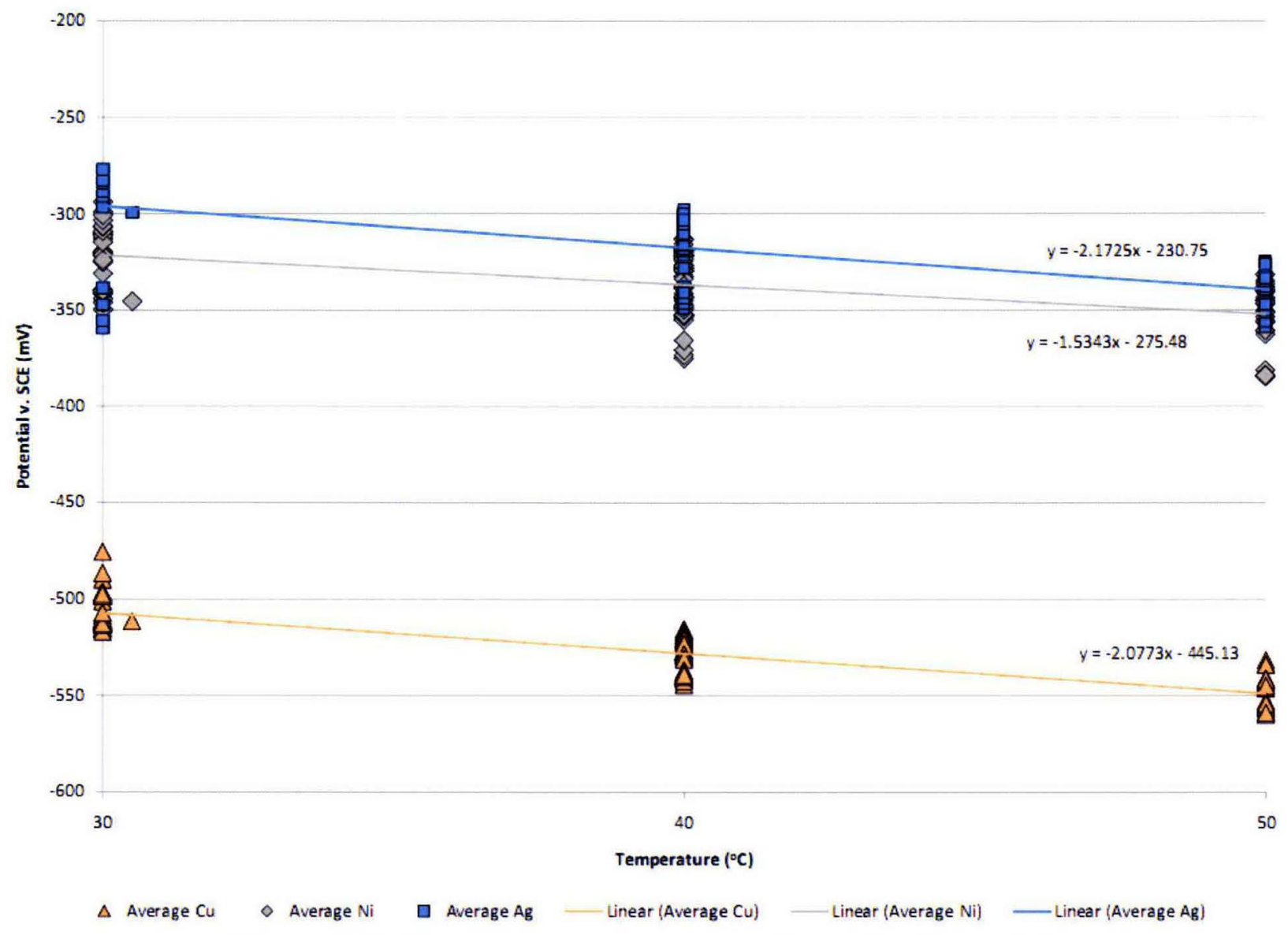

Figure 3-5. Temperature vs. Average Corrosion Potentials.

\subsection{CONCLUSIONS}

Based on the test data, future tank and tank steel electrode corrosion potential measurements can be made relative to the secondary reference electrodes, but these measurements should be corrected for changes in tank waste temperature. The correction factor is approximately $2 \mathrm{mV}$ per degree Celsius of temperature difference, where temperature difference is defined as the difference between tank temperature at the time of measurement and $30^{\circ} \mathrm{C}$, the average tank temperature during the first several months of 241-AN-102 MPCMS operation (when the corrosion potentials of the secondary reference electrodes were being recorded relative to the primary reference electrodes).

\subsection{REFERENCES}

RPP-PLAN-24604, 2007, Test Plan for Evaluating Secondary Reference Electrode Materials for the 241-AN-102 and the 241-AY-102 Multi-Probe Corrosion Monitoring Systems, Rev. 0, CH2M HILL Hanford Group, Inc., Richland, Washington.

RPP-SPEC-32496, 2007, Procurement Specification for AN-102 Corrosion Probe Assembly, Rev. 1, CH2M HILL Hanford Group, Inc., Richland, Washington. 
RPP-RPT-38931, Rev. 0

APPENDIX A

241-AN-102 "PRESENT" SIMULANT COMPOSITION 
RPP-RPT-38931, Rev. 0

\section{1-AN-102 "PRESENT" SIMULANT (TARGET VALUES)}

\begin{tabular}{|l|c|}
\hline Analyte/lon & Molarity (M) \\
\hline Antimony & $9.198 \mathrm{E}-05$ \\
\hline Barium & $5.689 \mathrm{E}-06$ \\
\hline Bismuth & $7.940 \mathrm{E}-04$ \\
\hline Cadmium & $5.326 \mathrm{E}-04$ \\
\hline Calcium & $1.059 \mathrm{E}-02$ \\
\hline Cerium & $3.881 \mathrm{E}-04$ \\
\hline Cobalt & $1.744 \mathrm{E}-04$ \\
\hline Copper & $3.702 \mathrm{E}-04$ \\
\hline Europium & $8.338 \mathrm{E}-07$ \\
\hline Iron & $5.967 \mathrm{E}-04$ \\
\hline Lanthanum & $1.043 \mathrm{E}-04$ \\
\hline Lead & $8.014 \mathrm{E}-04$ \\
\hline Manganese & $3.379 \mathrm{E}-04$ \\
\hline Neodymium & $1.809 \mathrm{E}-04$ \\
\hline Nickel & $6.757 \mathrm{E}-03$ \\
\hline Potassium & $5.380 \mathrm{E}-02$ \\
\hline Samarium & $4.806 \mathrm{E}-05$ \\
\hline Silver & $1.241 \mathrm{E}-04$ \\
\hline Strontium & $2.636 \mathrm{E}-05$ \\
\hline Vanadyl & $4.229 \mathrm{E}-05$ \\
\hline Yttrium & $2.170 \mathrm{E}-05$ \\
\hline Zinc & $4.713 \mathrm{E}-05$ \\
\hline Zirconyl & $8.097 \mathrm{E}-05$ \\
\hline
\end{tabular}

\begin{tabular}{|l|r|}
\hline Analyte/lon & Molarity (M) \\
\hline Aluminate & $4.923 \mathrm{E}-01$ \\
\hline Borate & $3.778 \mathrm{E}-03$ \\
\hline Chloride & $1.029 \mathrm{E}-01$ \\
\hline Chromate & $4.564 \mathrm{E}-03$ \\
\hline Molybdate & $5.221 \mathrm{E}-04$ \\
\hline Phosphate & $5.760 \mathrm{E}-02$ \\
\hline Silicate & $1.327 \mathrm{E}-03$ \\
\hline Sulfate & $1.395 \mathrm{E}-01$ \\
\hline Carbonate & $1.136 \mathrm{E}+00$ \\
\hline Hydroxide & $5.183 \mathrm{E}-01$ \\
\hline Nitrate & $3.192 \mathrm{E}+00$ \\
\hline Nitrite & $1.822 \mathrm{E}+00$ \\
\hline Acetate & $1.640 \mathrm{E}-02$ \\
\hline Formate & $2.133 \mathrm{E}-01$ \\
\hline Glycolate & $1.695 \mathrm{E}-01$ \\
\hline Oxalate & $8.400 \mathrm{E}-03$ \\
\hline Citrate & $8.380 \mathrm{E}-02$ \\
\hline EDTA & $3.620 \mathrm{E}-02$ \\
\hline HEDTA & $1.430 \mathrm{E}-02$ \\
\hline NTA & $5.700 \mathrm{E}-03$ \\
\hline IDA & $8.470 \mathrm{E}-02$ \\
\hline Gluconate & $1.800 \mathrm{E}-02$ \\
\hline
\end{tabular}




\section{1-AN-102 "PRESENT" SIMULANT (AS-MIXED)}

\begin{tabular}{|c|c|c|c|}
\hline Simulant Source & Formula & Formula Weight & Molarity (M) \\
\hline Antimony trichloride & $\mathrm{SbCl}_{3}$ & 228.11 & $9.20 \mathrm{E}-05$ \\
\hline Barium nitrate & $\mathrm{Ba}\left(\mathrm{NO}_{3}\right)_{2}$ & 261.38 & 5.69E-06 \\
\hline Bismuth nitrate, 5-hydrate & $\mathrm{Bi}\left(\mathrm{NO}_{3}\right)_{2}\left(5 \mathrm{H}_{2} \mathrm{O}\right)$ & 485.07 & 7.94E-04 \\
\hline Boric acid & $\mathrm{H}_{3} \mathrm{BO}_{3}$ & 61.8 & $3.78 \mathrm{E}-03$ \\
\hline Cadmium nitrate, 4-hydrate & $\mathrm{Cd}\left(\mathrm{NO}_{3}\right)_{2}\left(4 \mathrm{H}_{2} \mathrm{O}\right)$ & 308.47 & 5.33E-04 \\
\hline Calcium nitrate, 4-hydrate & $\mathrm{Ca}\left(\mathrm{NO}_{3}\right)_{2}\left(4 \mathrm{H}_{2} \mathrm{O}\right)$ & 236.15 & 1.06E-02 \\
\hline Cerous nitrate, 6 -hydrate & $\mathrm{Ce}\left(\mathrm{NO}_{3}\right)_{3}\left(6 \mathrm{H}_{2} \mathrm{O}\right)$ & 434.23 & $3.88 \mathrm{E}-04$ \\
\hline Sodium chromate, 4-hydrate & $\mathrm{Na}_{2} \mathrm{CrO}_{4}\left(4 \mathrm{H}_{2} \mathrm{O}\right)$ & 234.03 & 4.56E-03 \\
\hline Cobaltous nitrate, 6 -hydrate & $\mathrm{Co}\left(\mathrm{NO}_{3}\right)_{2}\left(6 \mathrm{H}_{2} \mathrm{O}\right)$ & 291 & 1.74E-04 \\
\hline Cupric nitrate, 2.5 hydrate & $\mathrm{Cu}\left(\mathrm{NO}_{3}\right)_{2}\left(2.5 \mathrm{H}_{2} \mathrm{O}\right)$ & 233 & $3.70 \mathrm{E}-04$ \\
\hline Europium nitrate, 6 -hydrate & $\mathrm{Eu}\left(\mathrm{NO}_{3}\right)_{3}\left(6 \mathrm{H}_{2} \mathrm{O}\right)$ & 446.07 & 8.34E-07 \\
\hline Ferric nitrate, 9-hydrate & $\mathrm{Fe}\left(\mathrm{NO}_{3}\right)_{3}\left(9 \mathrm{H}_{2} \mathrm{O}\right)$ & 404 & 5.97E-04 \\
\hline Lanthanum nitrate, 6-hydrate & $\mathrm{La}\left(\mathrm{NO}_{3}\right)_{3}\left(6 \mathrm{H}_{2} \mathrm{O}\right)$ & 433 & $1.04 \mathrm{E}-04$ \\
\hline Lead nitrate & $\mathrm{Pb}\left(\mathrm{NO}_{3}\right)_{2}$ & 331.21 & 8.01E-04 \\
\hline Manganous chloride, 4-hydrate & $\mathrm{MnCl}_{2}\left(4 \mathrm{H}_{2} \mathrm{O}\right)$ & 197.91 & 3.38E-04 \\
\hline Potassium molybdate & $\mathrm{K}_{2} \mathrm{MoO}_{4}$ & 238 & $5.22 \mathrm{E}-04$ \\
\hline Neodymium nitrate, 6-hydrate & $\mathrm{Nd}\left(\mathrm{NO}_{3}\right)_{3}\left(\mathrm{H}_{2} \mathrm{O}\right)$ & 438.35 & 1.81E-04 \\
\hline Nickel nitrate, 6-hydrate & $\mathrm{Ni}\left(\mathrm{NO}_{3}\right)_{2}\left(\mathrm{H}_{2} \mathrm{O}\right)$ & 290.81 & $6.76 \mathrm{E}-03$ \\
\hline Potassium nitrate & $\mathrm{K}\left(\mathrm{NO}_{3}\right)$ & 101.11 & 5.27E-02 \\
\hline Samarium nitrate, 6-hydrate & $\mathrm{Sm}\left(\mathrm{NO}_{3}\right)_{3}\left(6 \mathrm{H}_{2} \mathrm{O}\right)$ & 444.47 & 4.81E-05 \\
\hline Sodium silicate, 9-hydrate & $\mathrm{Na}_{2} \mathrm{SiO}_{3}\left(9 \mathrm{H}_{2} \mathrm{O}\right)$ & 284 & 1.33E-03 \\
\hline Silver nitrate & $\mathrm{AgNO}_{3}$ & 153.89 & 1.24E-04 \\
\hline Strontium nitrate & $\mathrm{Sr}\left(\mathrm{NO}_{3}\right)_{2}$ & 211.65 & 2.64E-05 \\
\hline Vanadyl sulfate, 2-hydrate & $\mathrm{VO}\left(\mathrm{SO}_{4}\right)\left(3 \mathrm{H}_{2} \mathrm{O}\right)$ & 217.11 & $4.23 E-05$ \\
\hline Yttrium nitrate, 6-hydrate & $\mathrm{Yt}\left(\mathrm{NO}_{3}\right)_{3}\left(6 \mathrm{H}_{2} \mathrm{O}\right)$ & 383.01 & 2.17E-05 \\
\hline Zinc nitrate, 6-hydrate & $\mathrm{Zn}\left(\mathrm{NO}_{3}\right)_{2}\left(6 \mathrm{H}_{2} \mathrm{O}\right)$ & 297.49 & 4.71E-05 \\
\hline Zirconyl nitrate, $x$-hydrate & $\mathrm{ZrO}\left(\mathrm{NO}_{3}\right)_{2}\left(\mathrm{xH}_{2} \mathrm{O}\right), \mathrm{x}=1$ & 249.224 & $8.10 \mathrm{E}-05$ \\
\hline Sodium chloride & $\mathrm{NaCl}$ & 58.44 & 1.03E-01 \\
\hline Sodium sulfate & $\mathrm{Na}_{2} \mathrm{SO}_{4}$ & 142.04 & $1.40 \mathrm{E}-01$ \\
\hline Glycolic acid & $\mathrm{C}_{2} \mathrm{H}_{4} \mathrm{O}_{3}$ & 76.05 & $1.70 \mathrm{E}-01$ \\
\hline Citric acid, 1-hydrate & $\mathrm{C}_{6} \mathrm{H}_{8} \mathrm{O}_{7}\left(\mathrm{H}_{2} \mathrm{O}\right)$ & 210.14 & $8.38 \mathrm{E}-02$ \\
\hline Disodium EDTA & $\mathrm{Na}_{2} \mathrm{C}_{10} \mathrm{H}_{14} \mathrm{O}_{8}\left(2 \mathrm{H}_{2} \mathrm{O}\right)$ & 372.24 & $3.62 \mathrm{E}-02$ \\
\hline HEDTA & $\mathrm{C}_{10} \mathrm{H}_{18} \mathrm{~N}_{2} \mathrm{O}_{7}$ & 278.26 & 1.43E-02 \\
\hline Nitrilotriacetic Acid & $\mathrm{C}_{6} \mathrm{H}_{9} \mathrm{NO}_{6}$ & 191.14 & 5.70E-03 \\
\hline Iminodiacetic Acid & $\mathrm{C}_{4} \mathrm{H}_{7} \mathrm{NO}_{2}$ & 133.1 & 8.47E-02 \\
\hline Sodium Gluconate & $\mathrm{C}_{6} \mathrm{H}_{11} \mathrm{O}_{7} \mathrm{Na}$ & 218.14 & $1.80 \mathrm{E}-02$ \\
\hline Sodium hydroxide & $\mathrm{NaOH}$ & 40 & $3.21 \mathrm{E}+00$ \\
\hline Aluminum nitrate, 9-hydrate & $\mathrm{Al}\left(\mathrm{NO}_{3}\right)_{3}\left(\mathrm{H}_{2} \mathrm{O}\right)$ & 375.13 & $4.92 E-01$ \\
\hline Sodium phosphate, 12-hydrate & $\mathrm{Na}_{3} \mathrm{PO}_{4}\left(12 \mathrm{H}_{2} \mathrm{O}\right)$ & 380.12 & $5.76 \mathrm{E}-02$ \\
\hline Sodium formate & $\mathrm{Na}\left(\mathrm{CHO}_{2}\right)$ & 68.01 & 2.13E-01 \\
\hline Sodium acetate, 3-hydrate & $\mathrm{Na}\left(\mathrm{C}_{2} \mathrm{H}_{3} \mathrm{O}_{2}\right)\left(3 \mathrm{H}_{2} \mathrm{O}\right)$ & 136.08 & $1.64 \mathrm{E}-02$ \\
\hline Sodium oxalate & $\mathrm{Na}_{2}\left(\mathrm{C}_{2} \mathrm{O}_{4}\right)$ & 134 & $8.40 \mathrm{E}-03$ \\
\hline Sodium carbonate & $\mathrm{Na}_{2}\left(\mathrm{CO}_{3}\right)$ & 106 & $1.14 \mathrm{E}+00$ \\
\hline Sodium nitrate & $\mathrm{NaNO}_{3}$ & 84.99 & $1.61 \mathrm{E}+00$ \\
\hline Sodium nitrite & $\mathrm{NaNO}_{2}$ & 69 & $1.82 \mathrm{E}+00$ \\
\hline
\end{tabular}


RPP-RPT-38931, Rev. 0

APPENDIX B

241-AN-102 “10-YEAR" SIMULANT COMPOSITION

B-1 
RPP-RPT-38931, Rev. 0

241-AN-102 “10-YEAR” SIMULANT (TARGET VALUES)

\begin{tabular}{|l|c|}
\hline Analyte/lon & Molarity (M) \\
\hline Antimony & $9.198 \mathrm{E}-05$ \\
\hline Barium & $5.689 \mathrm{E}-06$ \\
\hline Bismuth & $7.940 \mathrm{E}-04$ \\
\hline Cadmium & $5.326 \mathrm{E}-04$ \\
\hline Calcium & $1.059 \mathrm{E}-02$ \\
\hline Cerium & $3.881 \mathrm{E}-04$ \\
\hline Cobalt & $1.744 \mathrm{E}-04$ \\
\hline Copper & $3.702 \mathrm{E}-04$ \\
\hline Europium & $8.338 \mathrm{E}-07$ \\
\hline Iron & $5.967 \mathrm{E}-04$ \\
\hline Lanthanum & $1.043 \mathrm{E}-04$ \\
\hline Lead & $8.014 \mathrm{E}-04$ \\
\hline Manganese & $3.379 \mathrm{E}-04$ \\
\hline Neodymium & $1.809 \mathrm{E}-04$ \\
\hline Nickel & $6.757 \mathrm{E}-03$ \\
\hline Potassium & $5.380 \mathrm{E}-02$ \\
\hline Samarium & $4.806 \mathrm{E}-05$ \\
\hline Silver & $1.241 \mathrm{E}-04$ \\
\hline Strontium & $2.636 \mathrm{E}-05$ \\
\hline Vanadyl & $4.229 \mathrm{E}-05$ \\
\hline Yttrium & $2.170 \mathrm{E}-05$ \\
\hline Zinc & $4.713 \mathrm{E}-05$ \\
\hline Zirconyl & $8.097 \mathrm{E}-05$ \\
\hline &
\end{tabular}

\begin{tabular}{|l|c|}
\hline Analyte/lon & Molarity (M) \\
\hline Aluminate & $4.923 \mathrm{E}-01$ \\
\hline Borate & $3.778 \mathrm{E}-03$ \\
\hline Chloride & $1.029 \mathrm{E}-01$ \\
\hline Chromate & $4.564 \mathrm{E}-03$ \\
\hline Molybdate & $5.221 \mathrm{E}-04$ \\
\hline Phosphate & $5.760 \mathrm{E}-02$ \\
\hline Silicate & $1.327 \mathrm{E}-03$ \\
\hline Sulfate & $1.395 \mathrm{E}-01$ \\
\hline Carbonate & $1.61 \mathrm{E}+00$ \\
\hline Hydroxide & $1.81 \mathrm{E}-01$ \\
\hline Nitrate & $2.88 \mathrm{E}+00$ \\
\hline Nitrite & $2.26 \mathrm{E}+00$ \\
\hline Acetate & $1.25 \mathrm{E}-02$ \\
\hline Formate & $1.62 \mathrm{E}-01$ \\
\hline Glycolate & $1.29 \mathrm{E}-01$ \\
\hline Oxalate & $6.37 \mathrm{E}-03$ \\
\hline Citrate & $6.37 \mathrm{E}-02$ \\
\hline EDTA & $2.75 \mathrm{E}-02$ \\
\hline HEDTA & $1.09 \mathrm{E}-02$ \\
\hline NTA & $4.34 \mathrm{E}-03$ \\
\hline IDA & $6.44 \mathrm{E}-02$ \\
\hline Gluconate & $1.370 \mathrm{E}-02$ \\
\hline
\end{tabular}


241-AN-102 “10-YEAR" SIMULANT (AS-MIXED)

\begin{tabular}{|c|c|c|c|}
\hline Simulant Source & Formula & Formula Weight & Molarity (M) \\
\hline Antimony trichloride & $\mathrm{SbCl}_{3}$ & 228.11 & $9.20 \mathrm{E}-05$ \\
\hline Barium nitrate & $\mathrm{Ba}\left(\mathrm{NO}_{3}\right)_{2}$ & 261.38 & $5.69 \mathrm{E}-06$ \\
\hline Bismuth nitrate, 5-hydrate & $\mathrm{Bi}\left(\mathrm{NO}_{3}\right)_{2}\left(5 \mathrm{H}_{2} \mathrm{O}\right)$ & 485.07 & 7.94E-04 \\
\hline Boric acid & $\mathrm{H}_{3} \mathrm{BO}_{3}$ & 61.8 & 3.78E-03 \\
\hline Cadmium nitrate, 4-hydrate & $\mathrm{Cd}\left(\mathrm{NO}_{3}\right)_{2}\left(4 \mathrm{H}_{2} \mathrm{O}\right)$ & 308.47 & 5.33E-04 \\
\hline Calcium nitrate, 4-hydrate & $\mathrm{Ca}\left(\mathrm{NO}_{3}\right)_{2}\left(4 \mathrm{H}_{2} \mathrm{O}\right)$ & 236.15 & 1.06E-02 \\
\hline Cerous nitrate, 6-hydrate & $\mathrm{Ce}\left(\mathrm{NO}_{3}\right)_{3}\left(6 \mathrm{H}_{2} \mathrm{O}\right)$ & 434.23 & $3.88 E-04$ \\
\hline Sodium chromate, 4-hydrate & $\mathrm{Na}_{2} \mathrm{CrO}_{4}\left(4 \mathrm{H}_{2} \mathrm{O}\right)$ & 234.03 & $4.56 \mathrm{E}-03$ \\
\hline Cobaltous nitrate, 6 -hydrate & $\mathrm{Co}\left(\mathrm{NO}_{3}\right)_{2}\left(6 \mathrm{H}_{2} \mathrm{O}\right)$ & 291 & $1.74 \mathrm{E}-04$ \\
\hline Cupric nitrate, 2.5 hydrate & $\mathrm{Cu}\left(\mathrm{NO}_{3}\right)_{2}\left(2.5 \mathrm{H}_{2} \mathrm{O}\right)$ & 233 & 3.70E-04 \\
\hline Europium nitrate, 6 -hydrate & $\mathrm{Eu}\left(\mathrm{NO}_{3}\right)_{3}\left(6 \mathrm{H}_{2} \mathrm{O}\right)$ & 446.07 & 8.34E-07 \\
\hline Ferric nitrate, 9-hydrate & $\mathrm{Fe}\left(\mathrm{NO}_{3}\right)_{3}\left(9 \mathrm{H}_{2} \mathrm{O}\right)$ & 404 & 5.97E-04 \\
\hline Lanthanum nitrate, 6-hydrate & $\mathrm{La}\left(\mathrm{NO}_{3}\right)_{3}\left(6 \mathrm{H}_{2} \mathrm{O}\right)$ & 433 & $1.04 \mathrm{E}-04$ \\
\hline Lead nitrate & $\mathrm{Pb}\left(\mathrm{NO}_{3}\right)_{2}$ & 331.21 & $8.01 \mathrm{E}-04$ \\
\hline Manganous chloride, 4-hydrate & $\mathrm{MnCl}_{2}\left(4 \mathrm{H}_{2} \mathrm{O}\right)$ & 197.91 & 3.38E-04 \\
\hline Potassium molybdate & $\mathrm{K}_{2} \mathrm{MoO}_{4}$ & 238 & 5.22E- 04 \\
\hline Neodymium nitrate, 6-hydrate & $\mathrm{Nd}\left(\mathrm{NO}_{3}\right)_{3}\left(\mathrm{H}_{2} \mathrm{O}\right)$ & 438.35 & $1.81 \mathrm{E}-04$ \\
\hline Nickel nitrate, 6-hydrate & $\mathrm{Ni}\left(\mathrm{NO}_{3}\right)_{2}\left(\mathrm{H}_{2} \mathrm{O}\right)$ & 290.81 & $6.76 \mathrm{E}-03$ \\
\hline Potassium nitrate & $\mathrm{K}\left(\mathrm{NO}_{3}\right)$ & 101.11 & $5.27 \mathrm{E}-02$ \\
\hline Samarium nitrate, 6-hydrate & $\mathrm{Sm}\left(\mathrm{NO}_{3}\right)_{3}\left(6 \mathrm{H}_{2} \mathrm{O}\right)$ & 444.47 & $4.81 \mathrm{E}-05$ \\
\hline Sodium silicate, 9-hydrate & $\mathrm{Na}_{2} \mathrm{SiO}_{3}\left(9 \mathrm{H}_{2} \mathrm{O}\right)$ & 284 & 1.33E-03 \\
\hline Silver nitrate & $\mathrm{AgNO}_{3}$ & 153.89 & $1.24 \mathrm{E}-04$ \\
\hline Strontium nitrate & $\mathrm{Sr}\left(\mathrm{NO}_{3}\right)_{2}$ & 211.65 & 2.64E-05 \\
\hline Vanadyl sulfate, 2-hydrate & $\mathrm{VO}\left(\mathrm{SO}_{4}\right)\left(3 \mathrm{H}_{2} \mathrm{O}\right)$ & 217.11 & 4.23E-05 \\
\hline Yttrium nitrate, 6-hydrate & $\mathrm{Yt}\left(\mathrm{NO}_{3}\right)_{3}\left(6 \mathrm{H}_{2} \mathrm{O}\right)$ & 383.01 & 2.17E-05 \\
\hline Zinc nitrate, 6-hydrate & $\mathrm{Zn}\left(\mathrm{NO}_{3}\right)_{2}\left(6 \mathrm{H}_{2} \mathrm{O}\right)$ & 297.49 & 4.71E-05 \\
\hline Zirconyl nitrate, $x$-hydrate & $\mathrm{ZrO}\left(\mathrm{NO}_{3}\right)_{2}\left(\mathrm{xH}_{2} \mathrm{O}\right), \mathrm{x}=1$ & 249.224 & $8.10 \mathrm{E}-05$ \\
\hline Sodium chloride & $\mathrm{NaCl}$ & 58.44 & $1.02 \mathrm{E}-01$ \\
\hline Sodium sulfate & $\mathrm{Na}_{2} \mathrm{SO}_{4}$ & 142.04 & $1.40 \mathrm{E}-01$ \\
\hline Glycolic acid & $\mathrm{C}_{2} \mathrm{H}_{4} \mathrm{O}_{3}$ & 76.05 & $1.26 \mathrm{E}-01$ \\
\hline Citric acid, 1-hydrate & $\mathrm{C}_{6} \mathrm{H}_{8} \mathrm{O}_{7}\left(\mathrm{H}_{2} \mathrm{O}\right)$ & 210.14 & $6.21 \mathrm{E}-02$ \\
\hline Disodium EDTA & $\mathrm{Na}_{2} \mathrm{C}_{10} \mathrm{H}_{14} \mathrm{O}_{8}\left(2 \mathrm{H}_{2} \mathrm{O}\right)$ & 372.24 & $2.68 \mathrm{E}-02$ \\
\hline HEDTA & $\mathrm{C}_{10} \mathrm{H}_{18} \mathrm{~N}_{2} \mathrm{O}_{7}$ & 278.26 & $1.06 \mathrm{E}-02$ \\
\hline Nitrilotriacetic Acid & $\mathrm{C}_{6} \mathrm{H}_{9} \mathrm{NO}_{6}$ & 191.14 & $4.20 \mathrm{E}-03$ \\
\hline Iminodiacetic Acid & $\mathrm{C}_{4} \mathrm{H}_{7} \mathrm{NO}_{2}$ & 133.1 & $6.27 \mathrm{E}-02$ \\
\hline Sodium Gluconate & $\mathrm{C}_{6} \mathrm{H}_{11} \mathrm{O}_{7} \mathrm{Na}$ & 218.14 & $2.00 \mathrm{E}-02$ \\
\hline Sodium hydroxide & $\mathrm{NaOH}$ & 40 & $2.70 \mathrm{E}+00$ \\
\hline Aluminum nitrate, 9-hydrate & $\mathrm{Al}\left(\mathrm{NO}_{3}\right)_{3}\left(\mathrm{H}_{2} \mathrm{O}\right)$ & 375.13 & $4.92 \mathrm{E}-01$ \\
\hline Sodium phosphate, 12-hydrate & $\mathrm{Na}_{3} \mathrm{PO}_{4}\left(12 \mathrm{H}_{2} \mathrm{O}\right)$ & 380.12 & $5.76 \mathrm{E}-02$ \\
\hline Sodium formate & $\mathrm{Na}\left(\mathrm{CHO}_{2}\right)$ & 68.01 & $1.58 \mathrm{E}-01$ \\
\hline Sodium acetate, 3-hydrate & $\mathrm{Na}\left(\mathrm{C}_{2} \mathrm{H}_{3} \mathrm{O}_{2}\right)\left(3 \mathrm{H}_{2} \mathrm{O}\right)$ & 136.08 & $1.21 \mathrm{E}-02$ \\
\hline Sodium oxalate & $\mathrm{Na}_{2}\left(\mathrm{C}_{2} \mathrm{O}_{4}\right)$ & 134 & $6.20 \mathrm{E}-03$ \\
\hline Sodium carbonate & $\mathrm{Na}_{2}\left(\mathrm{CO}_{3}\right)$ & 106 & $1.61 \mathrm{E}+00$ \\
\hline Sodium nitrate & $\mathrm{NaNO}_{3}$ & 84.99 & $1.61 \mathrm{E}+00$ \\
\hline Sodium nitrite & $\mathrm{NaNO}_{2}$ & 69 & $1.82 E+00$ \\
\hline
\end{tabular}


RPP-RPT-38931, Rev. 0

\section{APPENDIX C}

241-AN-102 "20-YEAR" SIMULANT COMPOSITION

C-1 
241-AN-102 “20-YEAR” SIMULANT (TARGET VALUES)

\begin{tabular}{|l|r|}
\hline Analyte/lon & Molarity (M) \\
\hline Antimony & $9.198 \mathrm{E}-05$ \\
\hline Barium & $5.689 \mathrm{E}-06$ \\
\hline Bismuth & $7.940 \mathrm{E}-04$ \\
\hline Cadmium & $5.326 \mathrm{E}-04$ \\
\hline Calcium & $1.059 \mathrm{E}-02$ \\
\hline Cerium & $3.881 \mathrm{E}-04$ \\
\hline Cobalt & $1.744 \mathrm{E}-04$ \\
\hline Copper & $3.702 \mathrm{E}-04$ \\
\hline Europium & $8.338 \mathrm{E}-07$ \\
\hline Iron & $5.967 \mathrm{E}-04$ \\
\hline Lanthanum & $1.043 \mathrm{E}-04$ \\
\hline Lead & $8.014 \mathrm{E}-04$ \\
\hline Manganese & $3.379 \mathrm{E}-04$ \\
\hline Neodymium & $1.809 \mathrm{E}-04$ \\
\hline Nickel & $6.757 \mathrm{E}-03$ \\
\hline Potassium & $5.380 \mathrm{E}-02$ \\
\hline Samarium & $4.806 \mathrm{E}-05$ \\
\hline Silver & $1.241 \mathrm{E}-04$ \\
\hline Strontium & $2.636 \mathrm{E}-05$ \\
\hline Vanadyl & $4.229 \mathrm{E}-05$ \\
\hline Yttrium & $2.170 \mathrm{E}-05$ \\
\hline Zinc & $4.713 \mathrm{E}-05$ \\
\hline Zirconyl & $8.097 \mathrm{E}-05$ \\
\hline
\end{tabular}

\begin{tabular}{|l|r|}
\hline Analyte/lon & Molarity (M) \\
\hline Aluminate & $4.923 \mathrm{E}-01$ \\
\hline Borate & $3.778 \mathrm{E}-03$ \\
\hline Chloride & $1.029 \mathrm{E}-01$ \\
\hline Chromate & $4.564 \mathrm{E}-03$ \\
\hline Molybdate & $5.221 \mathrm{E}-04$ \\
\hline Phosphate & $5.760 \mathrm{E}-02$ \\
\hline Silicate & $1.327 \mathrm{E}-03$ \\
\hline Sulfate & $1.395 \mathrm{E}-01$ \\
\hline Carbonate & $2.136 \mathrm{E}+00$ \\
\hline Hydroxide & $6.300 \mathrm{E}-02$ \\
\hline Nitrate & $2.570 \mathrm{E}+00$ \\
\hline Nitrite & $2.700 \mathrm{E}+00$ \\
\hline Acetate & $8.530 \mathrm{E}-03$ \\
\hline Formate & $1.110 \mathrm{E}-01$ \\
\hline Glycolate & $8.820 \mathrm{E}-02$ \\
\hline Oxalate & $4.360 \mathrm{E}-03$ \\
\hline Citrate & $4.360 \mathrm{E}-02$ \\
\hline EDTA & $1.880 \mathrm{E}-02$ \\
\hline HEDTA & $7.430 \mathrm{E}-03$ \\
\hline NTA & $2.970 \mathrm{E}-03$ \\
\hline IDA & $4.410 \mathrm{E}-02$ \\
\hline Gluconate & $9.360 \mathrm{E}-03$ \\
\hline &
\end{tabular}


241-AN-102 “20-YEAR” SIMULANT (AS-MIXED)

\begin{tabular}{|c|c|c|c|}
\hline Simulant Source & Formula & Formula Weight & Molarity (M) \\
\hline Antimony trichloride & $\mathrm{SbCl}_{3}$ & 228.11 & $9.20 \mathrm{E}-05$ \\
\hline Barium nitrate & $\mathrm{Ba}\left(\mathrm{NO}_{3}\right)_{2}$ & 261.38 & 5.69E-06 \\
\hline Bismuth nitrate, 5-hydrate & $\mathrm{Bi}\left(\mathrm{NO}_{3}\right)_{2}\left(5 \mathrm{H}_{2} \mathrm{O}\right)$ & 485.07 & 7.94E-04 \\
\hline Boric acid & $\mathrm{H}_{3} \mathrm{BO}_{3}$ & 61.8 & $3.78 \mathrm{E}-03$ \\
\hline Cadmium nitrate, 4-hydrate & $\mathrm{Cd}\left(\mathrm{NO}_{3}\right)_{2}\left(4 \mathrm{H}_{2} \mathrm{O}\right)$ & 308.47 & 5.33E-04 \\
\hline Calcium nitrate, 4-hydrate & $\mathrm{Ca}\left(\mathrm{NO}_{3}\right)_{2}\left(4 \mathrm{H}_{2} \mathrm{O}\right)$ & 236.15 & $1.06 \mathrm{E}-02$ \\
\hline Cerous nitrate, 6 -hydrate & $\mathrm{Ce}\left(\mathrm{NO}_{3}\right)_{3}\left(6 \mathrm{H}_{2} \mathrm{O}\right)$ & 434.23 & $3.88 \mathrm{E}-04$ \\
\hline Sodium chromate, 4-hydrate & $\mathrm{Na}_{2} \mathrm{CrO}_{4}\left(4 \mathrm{H}_{2} \mathrm{O}\right)$ & 234.03 & $4.56 \mathrm{E}-03$ \\
\hline Cobaltous nitrate, 6 -hydrate & $\mathrm{Co}\left(\mathrm{NO}_{3}\right)_{2}\left(6 \mathrm{H}_{2} \mathrm{O}\right)$ & 291 & $1.74 \mathrm{E}-04$ \\
\hline Cupric nitrate, 2.5 hydrate & $\mathrm{Cu}\left(\mathrm{NO}_{3}\right)_{2}\left(2.5 \mathrm{H}_{2} \mathrm{O}\right)$ & 233 & $3.70 \mathrm{E}-04$ \\
\hline Europium nitrate, 6 -hydrate & $\mathrm{Eu}\left(\mathrm{NO}_{3}\right)_{3}\left(6 \mathrm{H}_{2} \mathrm{O}\right)$ & 446.07 & 8.34E-07 \\
\hline Ferric nitrate, 9-hydrate & $\mathrm{Fe}\left(\mathrm{NO}_{3}\right)_{3}\left(9 \mathrm{H}_{2} \mathrm{O}\right)$ & 404 & 5.97E-04 \\
\hline Lanthanum nitrate, 6-hydrate & $\mathrm{La}\left(\mathrm{NO}_{3}\right)_{3}\left(6 \mathrm{H}_{2} \mathrm{O}\right)$ & 433 & $1.04 \mathrm{E}-04$ \\
\hline Lead nitrate & $\mathrm{Pb}\left(\mathrm{NO}_{3}\right)_{2}$ & 331.21 & 8.01E-04 \\
\hline Manganous chloride, 4-hydrate & $\mathrm{MnCl}_{2}\left(4 \mathrm{H}_{2} \mathrm{O}\right)$ & 197.91 & $3.38 \mathrm{E}-04$ \\
\hline Potassium molybdate & $\mathrm{K}_{2} \mathrm{MoO}_{4}$ & 238 & $5.22 \mathrm{E}-04$ \\
\hline Neodymium nitrate, 6-hydrate & $\mathrm{Nd}\left(\mathrm{NO}_{3}\right)_{3}\left(\mathrm{H}_{2} \mathrm{O}\right)$ & 438.35 & 1.81E-04 \\
\hline Nickel nitrate, 6-hydrate & $\mathrm{Ni}\left(\mathrm{NO}_{3}\right)_{2}\left(\mathrm{H}_{2} \mathrm{O}\right)$ & 290.81 & $6.76 \mathrm{E}-03$ \\
\hline Potassium nitrate & $\mathrm{K}\left(\mathrm{NO}_{3}\right)$ & 101.11 & $5.27 \mathrm{E}-02$ \\
\hline Samarium nitrate, 6 -hydrate & $\mathrm{Sm}\left(\mathrm{NO}_{3}\right)_{3}\left(6 \mathrm{H}_{2} \mathrm{O}\right)$ & 444.47 & $4.81 \mathrm{E}-05$ \\
\hline Sodium silicate, 9-hydrate & $\mathrm{Na}_{2} \mathrm{SiO}_{3}\left(9 \mathrm{H}_{2} \mathrm{O}\right)$ & 284 & $1.33 \mathrm{E}-03$ \\
\hline Silver nitrate & $\mathrm{AgNO}_{3}$ & 153.89 & 1.24E-04 \\
\hline Strontium nitrate & $\mathrm{Sr}\left(\mathrm{NO}_{3}\right)_{2}$ & 211.65 & 2.64E-05 \\
\hline Vanadyl sulfate, 2-hydrate & $\mathrm{VO}\left(\mathrm{SO}_{4}\right)\left(3 \mathrm{H}_{2} \mathrm{O}\right)$ & 217.11 & 4.23E-05 \\
\hline Yttrium nitrate, 6-hydrate & $\mathrm{Yt}\left(\mathrm{NO}_{3}\right)_{3}\left(6 \mathrm{H}_{2} \mathrm{O}\right)$ & 383.01 & $2.17 E-05$ \\
\hline Zinc nitrate, 6-hydrate & $\mathrm{Zn}\left(\mathrm{NO}_{3}\right)_{2}\left(6 \mathrm{H}_{2} \mathrm{O}\right)$ & 297.49 & 4.71E-05 \\
\hline Zirconyl nitrate, $x$-hydrate & $\mathrm{ZrO}\left(\mathrm{NO}_{3}\right)_{2}\left(\mathrm{xH}_{2} \mathrm{O}\right), \mathrm{x}=1$ & 249.224 & 8.10E-05 \\
\hline Sodium chloride & $\mathrm{NaCl}$ & 58.44 & 1.02E-01 \\
\hline Sodium sulfate & $\mathrm{Na}_{2} \mathrm{SO}_{4}$ & 142.04 & 1.40E-01 \\
\hline Glycolic acid & $\mathrm{C}_{2} \mathrm{H}_{4} \mathrm{O}_{3}$ & 76.05 & 8.30E-02 \\
\hline Citric acid, 1-hydrate & $\mathrm{C}_{6} \mathrm{H}_{8} \mathrm{O}_{7}\left(\mathrm{H}_{2} \mathrm{O}\right)$ & 210.14 & 4.10E-02 \\
\hline Disodium EDTA & $\mathrm{Na}_{2} \mathrm{C}_{10} \mathrm{H}_{14} \mathrm{O}_{8}\left(2 \mathrm{H}_{2} \mathrm{O}\right)$ & 372.24 & 1.77E-02 \\
\hline HEDTA & $\mathrm{C}_{10} \mathrm{H}_{18} \mathrm{~N}_{2} \mathrm{O}_{7}$ & 278.26 & 7.00E-03 \\
\hline Nitrilotriacetic Acid & $\mathrm{C}_{6} \mathrm{H}_{9} \mathrm{NO}_{6}$ & 191.14 & $2.80 \mathrm{E}-03$ \\
\hline Iminodiacetic Acid & $\mathrm{C}_{4} \mathrm{H}_{7} \mathrm{NO}_{2}$ & 133.1 & 4.14E-02 \\
\hline Sodium Gluconate & $\mathrm{C}_{6} \mathrm{H}_{11} \mathrm{O}_{7} \mathrm{Na}$ & 218.14 & 2.00E-02 \\
\hline Sodium hydroxide & $\mathrm{NaOH}$ & 40 & $2.39 \mathrm{E}+00$ \\
\hline Aluminum nitrate, 9-hydrate & $\mathrm{Al}\left(\mathrm{NO}_{3}\right)_{3}\left(\mathrm{H}_{2} \mathrm{O}\right)$ & 375.13 & 4.92E-01 \\
\hline Sodium phosphate, 12 -hydrate & $\mathrm{Na}_{3} \mathrm{PO}_{4}\left(12 \mathrm{H}_{2} \mathrm{O}\right)$ & 380.12 & 5.76E-02 \\
\hline Sodium formate & $\mathrm{Na}\left(\mathrm{CHO}_{2}\right)$ & 68.01 & 1.04E-01 \\
\hline Sodium acetate, 3-hydrate & $\mathrm{Na}\left(\mathrm{C}_{2} \mathrm{H}_{3} \mathrm{O}_{2}\right)\left(3 \mathrm{H}_{2} \mathrm{O}\right)$ & 136.08 & $8.00 \mathrm{E}-03$ \\
\hline Sodium oxalate & $\mathrm{Na}_{2}\left(\mathrm{C}_{2} \mathrm{O}_{4}\right)$ & 134 & $4.10 \mathrm{E}-03$ \\
\hline Sodium carbonate & $\mathrm{Na}_{2}\left(\mathrm{CO}_{3}\right)$ & 106 & $2.14 \mathrm{E}+00$ \\
\hline Sodium nitrate & $\mathrm{NaNO}_{3}$ & 84.99 & $9.96 \mathrm{E}-01$ \\
\hline Sodium nitrite & $\mathrm{NaNO}_{2}$ & 69 & $2.70 \mathrm{E}+00$ \\
\hline
\end{tabular}

